\title{
Some identities of degenerate Fubini polynomials arising from differential equations
}

\section{Sung-Soo Pyo}

Department of Mathematics Education, Silla University, Busan, Republic of Korea.

Communicated by S.-H. Rim

\begin{abstract}
Recently, Kim et al. have studied degenerate Fubini polynomials in [T. Kim, D. V. Dolgy, D. S. Kim, J. J. Seo, J. Nonlinear Sci. Appl., 9 (2016), 2857-2864]. Jang and Kim presented some identities of Fubini polynomials arising from differential equations in [G.-W. Jang, T. Kim, Adv. Studies Contem. Math., 28 (2018), to appear]. In this paper, we drive differential equations from the generating function of the degenerate Fubini polynomials. In addition, we obtain some identities from those differential equations.
\end{abstract}

Keywords: Differential equations, Fubini polynomials, degenerate Fubini polynomials.

2010 MSC: 11B68, 11B83, 42A16.

(C)2018 All rights reserved.

\section{Introduction}

As is well known that the Fubini polynomials $\mathrm{F}_{\mathrm{n}}(\mathrm{y})$ are defined by the generating function to be

$$
\frac{1}{1-y\left(e^{t}-1\right)}=\sum_{n=0}^{\infty} F_{n}(y) \frac{t^{n}}{n !} \text {. }
$$

In the spacial case $y=1$,

$$
\frac{1}{2-e^{t}}=\sum_{n=0}^{\infty} F_{n}(1) \frac{t^{n}}{n !}=\sum_{n=0}^{\infty} F_{n} \frac{t^{n}}{n !}=\sum_{n=0}^{\infty} \sum_{k=0}^{n} k ! S_{2}(n, k) \frac{t^{n}}{n !},
$$

where $S_{2}(n, k)$ denotes the Stirling numbers of the second kind and $F_{n}(1)=F_{n}$ are called the Fubini numbers or the ordered Bell numbers.

From the definition of the generating function of Fubini polynomials, the equation (1.1), we obtain

$$
\frac{1}{1-y\left(e^{t}-1\right)}=\sum_{k=0}^{\infty} y^{k}\left(e^{t}-1\right)^{k}=\sum_{k=0}^{\infty} y^{k} k ! \frac{\left(e^{t}-1\right)^{k}}{k !}=\sum_{n=0}^{\infty} \sum_{k=0}^{n} k ! S_{2}(n, k) y^{k} \frac{t^{k}}{k !} \text {. }
$$

Email address: ssoopyo@silla.ac.kr (Sung-Soo Pyo)

doi: $10.22436 /$ jnsa.011.03.07

Received: 2017-12-25 Revised: 2018-01-17 Accepted: 2018-01-20 
The equations (1.1) and (1.2) indicate that

$$
F_{n}(y)=\sum_{k=0}^{n} k ! S_{2}(n, k) y^{k} .
$$

For a nonnegative integer $n$ and any real $x$, the symbol $(x)_{n}$ denotes the falling factorials, that is,

$$
(x)_{0}=1,(x)_{n}=x(x-1)(x-2) \cdots(x-n+1) .
$$

In [7], Kim introduced $\lambda$-analogue falling factorials and presented several results regarding it. The $\lambda$ analogue of falling factorials are defined as follows:

$$
(x)_{0, \lambda}=1,(x)_{n, \lambda}=x(x-\lambda)(x-2 \lambda) \cdots(x-(n-1) \lambda) .
$$

The degenerate Stirling numbers of the second kind are given by the generating function

$$
\frac{1}{k !}\left((1+\lambda t)^{\frac{1}{\lambda}}-1\right)^{k}=\sum_{n=k}^{\infty} S_{2, \lambda}(n, k) \frac{t^{n}}{n !} .
$$

It is well known that the Fubini numbers count the number of weak orderings on a set of $n$ elements [2]. Velleman and Call consider combination locks with a numeric keypad [23]. Pippenger traces the problem of counting weak orderings to the work of Whitworth [19]. Recently, several authors have studied Fubini numbers and polynomials (see $[3,5,18]$ ).

In [1], Carlitz presented degenerate Stirling, Bernoulli, and Eulerian numbers. After Carlitz, a group of mathematician have studied the degenerate special numbers. For example, degenerate Fubini polynomials are studied in [11], degenerated Bell polynomials in [14], degenerate Cauchy numbers in [20, 21], and degenerate Daehee numbers in [16, 22]. Lim studied degenerate Gennochi polynomials [17].

In [11], Kim et al. introduced the degenerate Fubini polynomials as follows:

$$
\frac{1}{1-y\left((1+\lambda t)^{\frac{1}{\lambda}}-1\right)}=\sum_{n=0}^{\infty} F_{n, \lambda}(y) \frac{t^{n}}{n !} .
$$

Note that $\lim _{\lambda \rightarrow 0} F_{n, \lambda}=F_{n}(y)$.

Several recurrence relations and some properties of the degenerate Fubini polynomials are presented, for example,

$$
F_{n, \lambda}(y)=\sum_{k=0}^{n} y^{k} k ! S_{2, \lambda}(n, k),
$$

where $S_{2, \lambda}(n, k)$ denotes the degenerate Stirling numbers of the second kind [11].

Recently, many mathematicians have studied special numbers using differential equations. Bernoulli numbers of the second kind are presented in [9], Frobenius-Euler polynomials are presented in [6], MittagLeffer polynomials are presented in [12], Changhee numbers and polynomials are presented in [8, 10], and Daehee and degenerate Daehee numbers are presented in [16, 20].

In this paper, we drive differential equations from the generating function of the degenerate Fubini polynomials. In addition, we obtain some identities from those differential equations.

\section{Preliminaries}

Throughout this article, for a positive integer $N$ and a function $Y$, we denote $Y^{N}$ for the $N$-th power of $Y$, and $Y^{(N)}$ for the $N$-th partial derivative of $Y$ with respect to $y$,

$$
Y^{N}=\underbrace{Y \times \cdots \times Y}_{N \text {-times }} \quad Y^{(0)}=Y, Y^{(N)}=\frac{\partial}{\partial y} Y^{(N-1)} .
$$


In [4], Jang and Kim presented some identities of Fubini polynomials arising from differential equations. They showed that the function $Y(t, y)=\frac{1}{1-y\left(e^{t}-1\right)}$ satisfies the following differential equation.

$$
\frac{\partial^{N} Y}{\partial t^{N}}=\sum_{k=0}^{N}(-1)^{N-k} a_{k}(N)(1+y)^{k} Y^{k+1}
$$

where $a_{0}(N)=1, a_{N}(N)=N$ !, and

$$
a_{k}(N)=\sum_{i_{1}=0}^{N-k} \sum_{i_{2}=0}^{i_{1}} \cdots \sum_{i_{k}=0}^{i_{k-1}}(k+1)^{N-k-i_{1}}\left(\prod_{j=1}^{k-1}(k-j+1)^{i_{j}-i_{j+1}+1}\right)
$$

for $1 \leqslant k \leqslant N-1$.

In this paper, the generating function of degenerate Fubini polynomials is used to derive some differential equations through the differentiation with respect to $y$.

For any positive integer $r$, the higher order degenerate Fubini polynomials, denoted by $F_{n, \lambda}^{o d}(r)$, are defined by the generating function to be:

$$
\left(\frac{1}{1-y\left((1+\lambda t)^{\frac{1}{\lambda}}-1\right)}\right)^{r}=\sum_{n=0}^{\infty} F_{n, \lambda}^{o d(r)}(y) \frac{t^{n}}{n !} .
$$

Let us consider the case when $r=2$.

$$
\begin{aligned}
\left(\frac{1}{1-y\left((1+\lambda t)^{\frac{1}{\lambda}}-1\right)}\right)^{2}=\left(\sum_{n=0}^{\infty} F_{n, \lambda}(y) \frac{t^{n}}{n !}\right)^{2} & =\left(\sum_{l_{2}=0}^{\infty} F_{l_{2}, \lambda}(y) \frac{t^{l_{2}}}{l_{2} !}\right)\left(\sum_{l_{1}=0}^{\infty} F_{l_{1}, \lambda}(y) \frac{t^{l_{1}}}{l_{1} !}\right) \\
& =\sum_{l_{2}=0}^{\infty} \sum_{l_{1}=0}^{l_{2}}\left(\begin{array}{l}
l_{2} \\
l_{1}
\end{array}\right) F_{l_{2}-l_{1}, \lambda}(y) F_{l_{1}, \lambda}(y) \frac{t^{l_{2}}}{l_{2} !} .
\end{aligned}
$$

From the previous equation (2.1) the following is obtained naturally.

$$
\sum_{n=0}^{\infty} F_{n, \lambda}^{o d(3)}(y) \frac{t^{n}}{n !}=\sum_{l_{3}=0}^{\infty} \sum_{l_{2}=0}^{l_{3}} \sum_{l_{1}=0}^{l_{2}}\left(\begin{array}{l}
l_{3} \\
l_{2}
\end{array}\right)\left(\begin{array}{l}
l_{2} \\
l_{1}
\end{array}\right) F_{l_{3}-l_{2}, \lambda}(y) F_{l_{2}-l_{1}, \lambda}(y) F_{l_{1}, \lambda}(y) \frac{t^{l_{3}}}{l_{3} !} .
$$

For brevity set $l_{0}=0$ and $l_{k}=n$. It is not difficult to obtain the following identity:

$$
\sum_{n=0}^{\infty} F_{n, \lambda}^{o d(k)}(y) \frac{t^{n}}{n !}=\sum_{n=0}^{\infty} \sum_{l_{k-1}=0}^{n} \cdots \sum_{l_{1}=0}^{l_{2}}\left(\prod_{i=0}^{k-1}\left(\begin{array}{c}
l_{i+1} \\
l_{i}
\end{array}\right) F_{l_{i+1}-l_{i}, \lambda}(y)\right) \frac{t^{n}}{n !} .
$$

The equation (2.2) yields an explicit formula for higher order Fubini polynomials.

Theorem 2.1. For any positive integer $k$, nonnegative integer $n$ and real $\lambda$,

$$
F_{n, \lambda}^{o d(k)}(y)=\sum_{l_{k-1}=0}^{n} \cdots \sum_{l_{1}=0}^{l_{2}}\left(\prod_{i=0}^{k-1}\left(\begin{array}{c}
l_{i+1} \\
l_{i}
\end{array}\right) F_{l_{i+1}-l_{i}, \lambda}(y)\right)
$$

where $l_{0}=0$ and $l_{\mathrm{k}}=\mathrm{n}$. holds.

In [5], some product of Fubini polynomials are considered. Kargin showed that following identity

$$
(y+1) \sum_{k=0}^{n}\left(\begin{array}{l}
n \\
k
\end{array}\right) F_{k}(y) F_{n-k}(y)=F_{n+1}(y)+F_{n}(y)(n \geqslant 0) .
$$

Here we obtain the $k$-th power of $F_{n, \lambda}(y)$. 
Theorem 2.2. For any positive integer $k$, nonnegative integer $n$, and real $\lambda$,

$$
F_{n, \lambda}^{k}(y)=\sum_{l_{k}=0}^{k n} \sum_{l_{k-1}=0}^{l_{k}} \cdots \sum_{l_{1}=0}^{l_{2}} l_{k} !\left(\prod_{i=0}^{k-1}\left(l_{i+1}-l_{i}\right) ! S_{2, \lambda}\left(n, l_{i+1}-l_{i}\right)\right) y^{l_{k}},
$$

where $l_{0}=0$.

Proof. From the expression (1.4), we get

$$
\begin{aligned}
\mathrm{F}_{n, \lambda}^{2}(y) & =\left(\sum_{l_{2}=0}^{n} l_{2} ! S_{2, \lambda}\left(n, l_{2}\right) y^{l_{2}}\right)\left(\sum_{l_{1}=0}^{n} l_{1} ! S_{2, \lambda}\left(n, l_{1}\right) y^{l_{1}}\right) \\
& =\sum_{l_{2}=0}^{2 n} \sum_{l_{1}=0}^{l_{2}}\left(l_{2}-l_{1}\right) ! l_{1} ! S_{2, \lambda}\left(n, l_{2}-l_{1}\right) S_{2, \lambda}\left(n, l_{1}\right) y^{l_{2}} .
\end{aligned}
$$

Let us assume that

$$
F_{n, \lambda}^{k-1}(y)=\sum_{l_{k-1}=0}^{(k-1) n} \sum_{l_{k-1}=0}^{l_{k}} \cdots \sum_{l_{1}=0}^{l_{2}}\left(\prod_{i=0}^{k-2}\left(l_{i+1}-l_{i}\right) ! S_{2, \lambda}\left(n, l_{i+1}-l_{i}\right)\right) y^{l_{k}},
$$

where $l_{0}=0$. Then we have the conclusion as follows:

$$
\begin{aligned}
F_{n, \lambda}^{k}(y) & =\left(\sum_{l_{k}=0}^{n} l_{k} ! S_{2, \lambda}\left(n, l_{k}\right) y^{l_{k}}\right) F_{n, \lambda}^{k-1}(y) \\
& =\left(\sum_{l_{k}=0}^{n} S_{2, \lambda}\left(n, l_{k}\right) y^{l_{3}}\right)\left(\sum_{l_{k-1}=0}^{(k-1) n} \cdots \sum_{l_{1}=0}^{l_{2}}\left(\prod_{i=0}^{k-2}\left(l_{i+1}-l_{i}\right) ! S_{2, \lambda}\left(n, l_{i+1}-l_{i}\right)\right) y^{l_{k-1}}\right) \\
& =\sum_{l_{k}=0}^{k n} \sum_{l_{k-1}=0}^{l_{k}} \cdots \sum_{l_{1}=0}^{l_{2}} l_{k} !\left(\prod_{i=0}^{k-1}\left(l_{i+1}-l_{i}\right) ! S_{2, \lambda}\left(n, l_{i+1}-l_{i}\right)\right) y^{l_{k}} .
\end{aligned}
$$

\section{Differential equations associated with the generating function of degenerate Fubini polynomials}

From now on, we use $Y$ to denote the generating function of the degenerate Fubini polynomials,

$$
Y=Y(y, t)=\frac{1}{1-y\left((1+\lambda t)^{\frac{1}{\lambda}}-1\right)} .
$$

By taking derivative with respect to $y$ of (3.1), we get

$$
Y^{(1)}=\frac{\partial Y}{\partial y}=\frac{\left((1+\lambda t)^{\frac{1}{\lambda}}-1\right)}{\left(1-y\left((1+\lambda t)^{\frac{1}{\lambda}}-1\right)\right)^{2}}=\frac{1-1+y\left((1+\lambda t)^{\frac{1}{\lambda}}-1\right)}{y\left(1-y\left((1+\lambda t)^{\frac{1}{\lambda}}-1\right)\right)^{2}}
$$

From (3.2), we have

$$
y Y^{(1)}=Y^{2}-Y
$$

Let us take derivative both sides of (3.3), and then multiply both sides by $y$, then we have

$$
y Y^{(1)}+y^{2} Y^{(2)}=2 y Y Y^{(1)}-y Y^{(1)}
$$

By (3.3) and (3.4), we get

$$
y^{2} Y^{(2)}=Y-Y^{2}+2 Y\left(Y^{2}-Y\right)-\left(Y^{2}-Y\right)=2 Y-3 Y^{2}+2 Y^{3} .
$$


One more time, differentiation of both sides of (3.5) and multiplying both sides by $y$, then we have:

$$
2 y^{2} Y^{(2)}+y^{3} Y^{(3)}=2 y Y^{(1)}-6 y Y Y^{(1)}+6 y Y^{2} Y^{(1)}
$$

From this, we get

$$
\begin{aligned}
y^{3} Y^{(3)} & =-2 y^{2} Y^{(2)}+2 y Y^{(1)}-6 y Y Y^{(1)}+6 y Y^{2} Y^{(1)} \\
& =-2\left(2 Y-3 Y^{2}+2 Y^{3}\right)+2\left(Y^{2}-Y\right)-6 Y\left(Y^{2}-Y\right)+6 Y^{2}\left(Y^{2}-Y\right) \\
& =-6 Y+14 Y^{2}-16 Y^{3}+6 Y^{4}
\end{aligned}
$$

To obtain $Y^{(\mathrm{N})}$, assume that

$$
y^{N} Y^{(N)}=\sum_{k=0}^{N} a_{k}(N) Y^{k+1}
$$

Let us take derivative of both sides of (3.6), and then multiply by $y$,

$$
N y^{N} Y^{(N)}+y^{N+1} Y^{(N+1)}=\sum_{k=0}^{N} a_{k}(N)(k+1) Y^{k} y Y^{(1)}
$$

By (3.3) and (3.7), we obtain

$$
\begin{aligned}
y^{N+1} Y^{(N+1)} & =-N \sum_{k=0}^{N} a_{k}(N) Y^{k+1}+\sum_{k=0}^{N} a_{k}(N)(k+1) Y^{k}\left(Y^{2}-Y\right) \\
& =-N \sum_{k=0}^{N} a_{k}(N) Y^{k}+\sum_{k=1}^{N+1} a_{k-1}(N) k Y^{k+1}-\sum_{k=0}^{N} a_{k}(N)(k+1) Y^{k+1} \\
& =\sum_{k=1}^{N}\left(-(N+k+1) a_{k}+k a_{k-1}(N)\right) Y^{k+1}-(N+1) a_{0}(N) Y+a_{N+1}(N)(N+1) Y^{N+2}
\end{aligned}
$$

Substituting $N+1$ instead of $N$ in (3.6), then the equation (3.6) becomes

$$
y^{N+1} Y^{(N+1)}=\sum_{k=0}^{N+1} a_{k}(N+1) Y^{k}
$$

Comparing coefficients on the both sides of (3.8) and (3.9), we get the following recurrence relations.

$$
\begin{aligned}
& a_{0}(N+1)=-(N+1) a_{0}(N), \quad a_{N+1}(N+1)=(N+1) a_{N+1}(N), \\
& a_{k}(N+1)=-(N+k+1) a_{k}(N)+k a_{k-1}(N) \text { for } 1 \leqslant k \leqslant N
\end{aligned}
$$

From (3.3), we know that

$$
a_{0}(1)=-1, \quad a_{1}(1)=1 .
$$

The recurrence relations (3.10) and initial conditions (3.11) yield the following:

$$
a_{0}(N)=(-1)^{N+1} N !, \quad a_{N}(N)=N !
$$


Let us observe the recurrence relation $a_{k}(N)(1 \leqslant k \leqslant N)$ with respect to $N$ in (3.10).

$$
\begin{aligned}
a_{k}(N)= & -(N+k) a_{k}(N-1)+k a_{k-1}(N-1) \\
= & -(N+k)\left(-(N+k-1) a_{k}(N-2)+k a_{k-1}(N-2)\right)+k a_{k-1}(N-1) \\
= & (N+k)(N+k-1) a_{k}(N-2)-k(N+k) a_{k-1}(N-2)+k a_{k-1}(N-1) \\
= & (N+k)(N+k-1)\left(-(N+k-2) a_{k}(N-3)+k a_{k-1}(N-3)\right) \\
& -k(N+k) a_{k-1}(N-2)+k a_{k-1}(N-1) \\
= & (-1)^{3}(N+k)_{3} a_{k}(N-3)+k(-1)^{2}(N+k)_{2} a_{k-1}(N-3) \\
& -k(N+k) a_{k-1}(N-2)+k a_{k-1}(N-1) \\
\vdots & (-1)^{N-k}(N+k)_{N-k} a_{k}(k) \\
& +\sum_{l_{1}=1}^{N-k-1}(-1)^{l_{1}} k(N+k)_{l_{1}} a_{k-1}\left(N-l_{1}-1\right)+k a_{k-1}(N-1) \\
= & \sum_{l_{1}=0}^{N-k}(-1)^{l_{1}} k(N+k)_{l_{1}} a_{k-1}\left(N-l_{1}-1\right) .
\end{aligned}
$$

Applying the processing in the equation (3.13) to $a_{k-1}\left(N-l_{1}-1\right)$,

$$
a_{k-1}\left(N-l_{1}-1\right)=\sum_{l_{2}=0}^{N-l_{1}-k}(-1)^{l_{2}}(k-1)\left(N-l_{1}+k-2\right)_{l_{2}} a_{k-2}\left(N-l_{1}-l_{2}-2\right) .
$$

For brevity set $\mathrm{N}_{0}=\mathrm{N}, \mathrm{N}_{\mathrm{i}}=\mathrm{N}-\sum_{\mathrm{m}=0}^{\mathrm{i}} \mathrm{l}_{\mathrm{i}}$ with $\mathrm{l}_{0}=0$, then we have

$$
\begin{aligned}
a_{k}\left(N_{0}-0\right) & =\sum_{l_{1}=0}^{N_{0}-k}(-1)^{l_{1}} k\left(N_{0}+k\right)_{1} a_{k-1}\left(N_{1}-1\right), \\
a_{k-1}\left(N_{1}-1\right) & =\sum_{l_{2}=0}^{N_{1}-k}(-1)^{l_{2}}(k-1)\left(N_{1}+k-2\right)_{l_{2}} a_{k-2}\left(N_{2}-2\right), \\
a_{k-2}\left(N_{2}-2\right) & =\sum_{l_{3}=0}^{N_{2}-k}(-1)^{l_{3}}(k-2)\left(N_{2}+k-4\right)_{l_{3}} a_{k-3}\left(N_{3}-3\right) .
\end{aligned}
$$

From (3.12) and $a_{0}\left(N_{k}-k\right)=(-1)^{N_{k}-k+1}\left(N_{k}-k\right)$ !, we get

$$
a_{k}(N)=\sum_{l_{1}=0}^{N_{0}-k} \sum_{l_{2}=0}^{N_{1}-k} \cdots \sum_{l_{k}=0}^{N_{k-1}-k}(-1)^{N-k+1} k !\left(N_{k}-k\right) ! \prod_{i=0}^{k}\left(N_{i}+k-2 i\right) l_{i},
$$

where $N_{0}=N, N_{i}=N-\sum_{m=0}^{i} l_{m}$, and $l_{0}=0$. We note that the coefficients $a_{k}(N)$ are similar to those in [10].

By (3.9) and (3.14), we obtain the following theorem.

Theorem 3.1. For $\mathrm{N} \in \mathbb{N}$, let us consider the following differential equation with respect to $\mathrm{y}$ :

$$
y^{N} Y^{(N)}=\sum_{k=0}^{N} a_{k}(N) Y^{k+1}
$$


where

$$
a_{k}(N)=\sum_{l_{1}=0}^{N_{0}-k} \sum_{l_{2}=0}^{N_{1}-k} \cdots \sum_{l_{k}=0}^{N_{k-1}-k}(-1)^{N-N_{k}} k !\left(N_{k}-k\right) ! \prod_{i=0}^{k}\left(N_{i}+k-2 i\right) l_{i}
$$

with $\mathrm{N}_{0}=\mathrm{N}, \mathrm{N}_{\mathrm{i}}=\mathrm{N}-\sum_{\mathrm{m}=0}^{\mathrm{i}} \mathrm{l}_{\mathrm{m}}, \mathrm{l}_{0}=0$, and $\mathrm{a}_{0}(\mathrm{~N})=(-1)^{\mathrm{N}+1} \mathrm{~N}$ !. Then $\mathrm{Y}=\frac{1}{1-\mathrm{y}\left((1+\lambda \mathrm{t})^{\frac{1}{\lambda}}-1\right)}$ is a solution of (3.15).

From (1.3), we have

$$
\begin{aligned}
Y^{(N)}=\frac{\partial^{N}}{\partial y^{N}}\left(\frac{1}{1-y\left((1+\lambda t)^{\frac{1}{\lambda}}-1\right)}\right) & =\frac{\partial^{N}}{\partial y^{N}} \sum_{n=0}^{\infty} F_{n, \lambda}(y) \frac{t^{n}}{n !} \\
& =\sum_{n=0}^{\infty} \frac{\partial^{N}}{\partial y^{N}}\left(\sum_{k=0}^{n} y^{k} k ! S_{2, \lambda}(n, k)\right) \frac{t^{n}}{n !} \\
& =\sum_{n=0}^{\infty} \sum_{k=N}^{n}(k)_{N} y^{k-N} k ! S_{2, \lambda}(n, k) \frac{t^{n}}{n !} .
\end{aligned}
$$

The equation (3.16) yields

$$
y^{N} Y^{(N)}=\sum_{n=0}^{\infty} \sum_{k=N}^{n}(k)_{N} y^{k} k ! S_{2, \lambda}(n, k) \frac{t^{n}}{n !}
$$

From the definition of $Y, Y^{k+1}$ is the generating function of higher-order Fubini polynomials. The higher-order Fubini polynomials expressed in (2.2).

$$
\begin{aligned}
\sum_{k=0}^{N} a_{k}(N) Y^{k+1} & =\sum_{k=0}^{N} a_{k}(N) \sum_{n=0}^{\infty} F_{n, \lambda}^{o d(k+1)}(y) \frac{t^{n}}{n !} \\
& =\sum_{k=0}^{N} a_{k}(N) \sum_{n=0}^{\infty}\left(\sum_{l_{k}=0}^{n} \cdots \sum_{l_{1}=0}^{l_{2}}\left(\prod_{i=0}^{k}\left(\begin{array}{c}
l_{i+1} \\
l_{i}
\end{array}\right) F_{l_{i+1}-l_{i}, \lambda}(y)\right)\right) \frac{t^{n}}{n !} \\
& =\sum_{k=0}^{N} \sum_{n=0}^{\infty} \sum_{l_{k}=0}^{n} \cdots \sum_{l_{1}=0}^{l_{2}} a_{k}(N)\left(\prod_{i=0}^{k}\left(\begin{array}{c}
l_{i+1} \\
l_{i}
\end{array}\right) F_{l_{i+1}-l_{i}, \lambda}(y)\right) \frac{t^{n}}{n !}
\end{aligned}
$$

By comparing the coefficients on the both sides in (3.17) and (3.18), we have the following identity.

Theorem 3.2. For any nonnegative integer $n$, positive integer $N$, and real $\lambda$,

$$
\sum_{k=0}^{n}(k)_{N} y^{k} k ! S_{2, \lambda}(n, k)=\sum_{k=0}^{N} \sum_{l_{k}=0}^{n} \ldots \sum_{l_{1}=0}^{l_{2}} a_{k}(N)\left(\prod_{i=1}^{k+1}\left(\begin{array}{c}
l_{i+1} \\
l_{i}
\end{array}\right) F_{l_{i}-l_{i-1}, \lambda}(y)\right),
$$

where

$$
a_{k}(N)=\sum_{l_{1}=0}^{N_{0}-k} \sum_{l_{2}=0}^{N_{1}-k} \cdots \sum_{l_{k}=0}^{N_{k-1}-k}(-1)^{N-N_{k}} k !\left(N_{k}-k\right) ! \prod_{i=0}^{k}\left(N_{i}+k-2 i\right) l_{i}
$$

with $\mathrm{N}_{0}=\mathrm{N}, \mathrm{N}_{\mathrm{i}}=\mathrm{N}-\sum_{\mathrm{m}=0}^{\mathrm{i}} \mathrm{l}_{\mathrm{m}}, \mathrm{l}_{0}=0$, and $\mathrm{a}_{0}(\mathrm{~N})=(-1)^{\mathrm{N}+1} \mathrm{~N}$ !.

Now we consider the inversion formula of Theorem 3.1. From (3.3), we have

$$
Y^{2}=Y+y Y^{(1)}
$$


Differentiating both sides of (3.19), then multiplying both sides by $y$, and then applying (3.3), we have

$$
2 Y\left(Y^{2}-Y\right)=2 y Y^{(1)}+y^{2} Y^{(2)} .
$$

Substituting $Y+y Y^{(1)}$ instead of $y^{2}$, the equation (3.20) becomes

$$
2 Y^{3}=2 Y^{2}+2 y Y^{(1)}+y^{2} Y^{(2)}=2\left(Y+y Y^{(1)}\right)+2 y Y^{(1)}+y^{2} Y^{(2)}=2 Y+4 y Y^{(1)}+y^{2} Y^{(2)}
$$

Continuing this process, we can set

$$
N ! Y^{N+1}=\sum_{k=0}^{N} b_{k}(N) y^{k} Y^{(k)}
$$

Let us take differentiate of both sides of (3.20), we have

$$
N !(N+1) Y^{N} Y^{(1)}=\sum_{k=0}^{N} b_{k}(N)\left(k y^{k-1} Y^{(k)}+y^{k} Y^{(k+1)}\right) .
$$

Multiply both sides by $y$, then we have

$$
\begin{aligned}
(N+1) ! Y^{N} y Y^{(1)} & =\sum_{k=0}^{N} b_{k}(N)\left(k y^{k-1} y Y^{(k)}+y^{k+1} Y^{(k+1)}\right) \\
& =\sum_{k=1}^{N} b_{k}(N) k y^{k} Y^{(k)}+\sum_{k=0}^{N} b_{k}(N) y^{k+1} Y^{(k+1)} \\
& =\sum_{k=1}^{N} b_{k}(N) k y^{k} Y^{(k)}+\sum_{k=1}^{N+1} b_{k-1}(N) y^{k} Y^{(k)}
\end{aligned}
$$

Since $y Y^{(1)}=Y^{2}-Y$, the equation (3.22) becomes

$$
\begin{aligned}
(N+1) ! Y^{N+2}= & (N+1) \sum_{k=0}^{N} b_{k}(N) y^{k} Y^{(k)}+\sum_{k=1}^{N} b_{k}(N) k y^{k} Y^{(k)}+\sum_{k=1}^{N+1} b_{k-1}(N) y^{k} Y^{(k)} \\
= & \sum_{k=1}^{N}\left((N+k+1) b_{k}(N)+b_{k-1}(N) y^{k}\right) Y^{(k)} \\
& +(N+1) b_{0}(N) Y+b_{N}(N) y^{N+1} Y^{(N+1)}
\end{aligned}
$$

Substituting $\mathrm{N}+1$ instead of $\mathrm{N}$ in (3.21),

$$
(N+1) ! Y^{N+2}=\sum_{k=0}^{N+1} b_{k}(N+1) y^{k} Y^{(k)} .
$$

Comparing the coefficients between (3.23) and (3.24), we get

$$
\begin{aligned}
b_{0}(N+1) & =(N+1) b_{0}(N), \quad b_{N+1}(N+1)=b_{N}(N), \\
b_{k}(N+1) & =(N+k+1) b_{k}(N)+b_{k-1}(N) \text { for } 1 \geqslant k \geqslant N .
\end{aligned}
$$

From (3.25) and $b_{0}(0)=b_{0}(1)=1$, we note that

$$
\mathrm{b}_{0}(\mathrm{~N})=\mathrm{N} !, \quad \mathrm{b}_{\mathrm{N}}(\mathrm{N})=\mathrm{b}_{\mathrm{N}-1}(\mathrm{~N}-1)=\cdots=\mathrm{b}_{0}(0)=1 .
$$


Let us observe the recurrence relation $b_{k}(N+1)$ with respect to $N$

$$
\begin{aligned}
b_{k}(N+1)= & (N+k+1) b_{k}(N)+b_{k-1}(N) \\
= & (N+k+1)\left((N+k) b_{k}(N-1)+b_{k-1}(N-1)\right)+b_{k-1}(N) \\
= & (N+k+1)_{2}\left((N+k-1) b_{k}(N-2)+b_{k-1}(N-2)\right) \\
& +(N+k+1) b_{k-1}(N-1)+b_{k-1}(N) \\
= & (N+k+1)_{3} b_{k}(N-2)+(N+k+1)_{2} b_{k-1}(N-2) \\
& +(N+k+1) b_{k-1}(N-1)+b_{k-1}(N) .
\end{aligned}
$$

Continuing this process, we have

$$
\begin{aligned}
b_{k}(N+1) & =(N+k+1)_{N-k+1} b_{k}(k)+\sum_{l_{1}=1}^{N-k}(N+k+1)_{l_{1}} b_{k-1}\left(N-l_{1}\right)+b_{k-1}(N) \\
& =\sum_{l_{1}=0}^{N-k+1}(N+k+1)_{l_{1}} b_{k-1}\left(N-l_{1}\right) .
\end{aligned}
$$

The recurrence relation (3.26) yields, for any positive integer $\mathrm{N}$ and nonnegative integer $\mathrm{k}$,

$$
b_{k}(N)=\sum_{l_{1}=0}^{N-k}(N+k) l_{1} b_{k-1}\left(N-l_{1}-1\right)
$$

From (3.27), we get

$$
\begin{aligned}
b_{k-1}\left(N-l_{1}-1\right) & =\sum_{l_{2}=0}^{N-l_{1}-k}\left(N-l_{1}+k-2\right)_{2} b_{k-2}\left(N-l_{1}-l_{2}-2\right), \\
b_{k-2}\left(N-l_{1}-l_{2}-2\right) & =\sum_{l_{3}=0}^{N-l_{1}-l_{2}-k}\left(N-l_{1}-l_{2}+k-4\right)_{l_{3}} b_{k-3}\left(N-l_{1}-l_{2}-l_{3}-3\right), \\
b_{0}\left(N-\sum_{i=1}^{k} l_{i}-k\right) & =\left(N-\sum_{i=1}^{k} l_{i}-k\right) !
\end{aligned}
$$

By substituting sequentially $b_{k}$, we obtain

$$
b_{k}(N)=\sum_{l_{1}=0}^{N_{1}-k} \sum_{l_{2}=0}^{N_{2}-k} \cdots \sum_{l_{k-1}=0}^{N_{k-1}-k}\left(N_{k}-k\right) !\left(\prod_{i=0}^{k-1}\left(N_{i}+k-2 i\right)_{l_{i}}\right),
$$

where

$$
\mathrm{N}_{\mathrm{i}}=\mathrm{N}-\sum_{\mathrm{m}=0}^{\mathrm{i}} l_{\mathrm{m}}, \quad \mathrm{N}_{0}=\mathrm{N}, \quad \text { and } \mathrm{l}_{0}=0 .
$$

By (3.21) and (3.28), we get the following theorem.

Theorem 3.3. For $\mathrm{N} \in \mathbb{N}$, let us consider the following differential equation with respect to $\mathrm{y}$ :

$$
N ! Y^{N+1}=\sum_{k=0}^{N} b_{k}(N) y^{k} Y^{(k)}
$$


where

$$
b_{k}(N)=\sum_{l_{1}=0}^{N_{1}-k} \sum_{l_{2}=0}^{N_{2}-k} \cdots \sum_{l_{k-1}=0}^{N_{k-1}-k}\left(N_{k}-k\right) !\left(\prod_{i=0}^{k-1}\left(N_{i}+k-2 i\right)_{l_{i}}\right)
$$

with

$$
\mathrm{N}_{\mathrm{i}}=\mathrm{N}-\sum_{\mathrm{m}=0}^{\mathrm{i}} l_{\mathrm{m}}, \mathrm{N}_{0}=\mathrm{N}, \text { and } \mathrm{l}_{0}=0
$$

Then $\mathrm{Y}=\frac{1}{1-\mathrm{y}\left((1+\lambda \mathrm{t})^{\frac{1}{\lambda}}-1\right)}$ is a solution of (3.29).

From (3.17), we know that

$$
y^{k} Y^{(k)}=\sum_{n=0}^{\infty} \sum_{m=k}^{n}(m)_{N} y^{m} m ! S_{2, \lambda}(n, m) \frac{t^{n}}{n !},
$$

and

$$
\sum_{k=0}^{N} b_{k}(N) y^{k} Y^{(k)}=\sum_{k=0}^{N} \sum_{n=0}^{\infty} \sum_{m=k}^{n} b_{k}(N)(m)_{k} y^{m} m ! S_{2, \lambda}(n, m) \frac{t^{n}}{n !} .
$$

From Theorem 2.1, we have

$$
Y^{N}=\sum_{n=0}^{\infty} F_{n, \lambda}^{o d(N)}(y) \frac{t^{n}}{n !}=\sum_{n=0}^{\infty} \sum_{l_{N-1}=0}^{n} \cdots \sum_{l_{1}=0}^{l_{2}}\left(\prod_{i=1}^{N}\left(\begin{array}{c}
l_{i+1} \\
l_{i}
\end{array}\right) F_{l_{i}-l_{i-1}, \lambda}(y)\right) \frac{t^{n}}{n !} .
$$

By (3.30) and (3.31), we have the following theorem.

Theorem 3.4. For any nonnegative integer $\mathrm{n}$ and positive integer $\mathrm{N}$,

$$
\sum_{n=0}^{\infty} \sum_{m=0}^{n} b_{k}(N)(m)_{k} y^{m} m ! S_{2, \lambda}(n, m)=\sum_{n=0}^{\infty} \sum_{l_{N-1}=0}^{n} \cdots \sum_{l_{1}=0}^{l_{2}}\left(\prod_{i=1}^{N}\left(\begin{array}{c}
l_{i+1} \\
l_{i}
\end{array}\right) F_{l_{i}-l_{i-1}, \lambda}(y)\right),
$$

where

$$
b_{k}(N)=\sum_{l_{1}=0}^{N_{1}-k} \sum_{l_{2}=0}^{N_{2}-k} \cdots \sum_{l_{k-1}=0}^{N_{k-1}-k}\left(N_{k}-k\right) !\left(\prod_{i=0}^{k-1}\left(N_{i}+k-2 i\right)_{l_{i}}\right)
$$

with

$$
\mathrm{N}_{\mathrm{i}}=\mathrm{N}-\sum_{\mathrm{m}=0}^{\mathrm{i}} l_{\mathrm{m}}, \mathrm{N}_{0}=\mathrm{N}, \text { and } \mathrm{l}_{0}=0
$$

\section{References}

[1] L. Carlitz, Degenerate Stirling, Bernoulli and Eulerian numbers, Utilitas Math., 15 (1979), 51-88. 1

[2] J.-M. De Koninck, Those Fascinating Numbers, American Mathematical Society, Providence, (2009). 1

[3] T. Diagana, H. Maïga, Some new identities and congruences for Fubini numbers, J. Numbers Theory, 173 (2017), $547-$ 569. 1

[4] G.-W. Jang, T. Kim, Some identities of Fubini polynomials arising from differential equations, Adv. Studies Contem. Math., 28 (2018), to appear. 2

[5] L. Kargin, Some formulae for products of Fubini polynomials with applications, Appl. Clas. Anal., arXiv preprint, (2016). 1,2

[6] T. Kim, Identities involving Frobenius-Euler polynomials arising from non-linear differential equations, J. Number Theory, 132 (2012), 2854-2865. 1

[7] T. Kim, A-analogue of Stirling numbers of the first kind, Adv. Stud. Contemp. Math., 27 (2017), 423-429. 1 
[8] T. Kim, D. V. Dolgy, D. S. Kim, J. J. Seo, Differential equations for Changhee polynomials and their applications, J. Nonlinear Sci. Appl., 9 (2016), 2857-2864. 1

[9] D. S. Kim, T. Kim, Some identities for Bernoulli numbers of the second kind arising from a nonlinear differential equation, Bull. Korean Math. Soc., 52 (2015), 2001-2010. 1

[10] T. Kim, D. S. Kim, A note on nonlinear Changhee differential equations, Russ. J. Math. Phys., 23 (2016), 88-92. 1, 3

[11] T. Kim, D. S. Kim, G.-W. Jang, A note on degenerate Fubini polynomials, Proc. Jangjeon. Math. Soc., 20 (2017), $521-531$. 1,1

[12] T. Kim, D. S. Kim, L. C. Jang, H. I. Kwon, Differential equations associated with Mittag-Leffer polynomials, Glob. J. Pure Appl. Math., 12 (2016), 2839-2847. 1

[13] S. Kim, B. M. Kim, J. Kwon, Differential equations associated with Genocchi polynomials, Glob. J. Pure Appl. Math., 12 (2016), 4579-4585.

[14] T. Kim, D. S. Kim, J. J. Seo, Differential equations associated with degenerate Bell polynomials, Inter. J. Pure Appl. Math., 108 (2016), 551-559. 1

[15] T. Kim, J. J. Seo, Revisit nonlinear differential equations arising from the generating functions of degenerate Bernoulli numbers, Adv. Stud. Contemp. Math., 26 (2016), 401-406.

[16] H. I. Kwon, T. Kim, J. J. Seo, A note on Daehee numbers arising from differential equations, Glob. J. Pure Appl., 12 (2016), 2349-2354. 1, 1

[17] D. Lim, Some identities of degenerate Genocchi polynomials, Bull. Korean Math. Soc., 53 (2016), 569-579. 1

[18] M. Muresan, G. Toader, A generalization of Fubini's number, Studia Univ. Babecş-Bolyai Math., 31 (1986), 60-65. 1

[19] N. Pippenger, The hypercube of resistors, asymptotic expansions, and preferential arrangements, Math. Mag., 83 (2010), 331-346. 1

[20] S.-S. Pyo, T. Kim, S.-H. Rim, Identities of the degenerate Daehee numbers with the Bernoulli numbers of the second kind arising from nonlinear Differential equation, J. Nonlinear Sci. Appl., 10 (2017), 6219-6228. 1, 1

[21] S.-S. Pyo, Degenerate Cauchy numbers and polynomials of the fourth kind, Adv. Studies. Contemp. Math., 28 (2018), to appear. 1

[22] S.-S. Pyo, T. Kim, S.-H. Rim, Degenerate Cauchy numbers of the third kind, preprint. 1

[23] D. J. Velleman, G. S. Call, Permutations and combination locks, Math. Mag., 68 (1995), 243-253. 1 